\title{
ISOMORPHISMS BETWEEN RADICAL WEIGHTED CONVOLUTION ALGEBRAS
}

\author{
by F. GHAHRAMANI
}

(Received 20th July 1982)

In [4] we have shown that any two semi-simple weighted convolution algebras $L^{1}\left(\omega_{1}\right)$ and $L^{1}\left(\omega_{2}\right)$ are isomorphic. In this paper, given any two radical weighted convolution algebras $L^{1}\left(\omega_{1}\right)$ and $L^{1}\left(\omega_{2}\right)$ we find necessary and sufficient conditions, in terms of $\omega_{1}$ and $\omega_{2}$, for $L^{1}\left(\omega_{1}\right)$ and $L^{1}\left(\omega_{2}\right)$ to be isomorphic.

We call a continuous and positive function $\omega$ on the non-negative real numbers $R^{+}$a weight function if $\omega(s+t) \leqq \omega(s) \omega(t)$ for every $s, t \in R^{+}$, and if $\omega(0)=1$. The weighted convolution algebra $L^{1}(\omega)$ is the (complex) Banach algebra of all equivalence classes of Lebesgue measurable functions $f$ such that $\|f\|=\int_{0}^{\infty}|f(t)| \omega(t) d t<\infty$, under pointwise addition, scalar multiplication of functions, and convolution product:

$$
(f * g)(x)=\int_{0}^{x} f(x-t) g(t) d t \quad\left(f, g \in L^{1}(\omega), \quad \text { a.e. } \quad x \in R^{+}\right)
$$

The elementary properties of the algebras $L^{1}(\omega)$ are given in [3]. We use the theory developed in [1], [2] and [4], and adopt the notation of [4].

We shall repeatedly use Titchmarsh's convolution theorem, which asserts that, if $\mu \neq 0$ and $v \neq 0$ are any two locally finite measures, then $\mu * v \neq 0$, or in its equivalent form $\alpha(\mu * v)=\alpha(\mu)+\alpha(v)$, where for every $\mu \neq 0, \alpha(\mu)$ is the infimum of the support of $\mu$ (see [1] for a proof).

If $\theta$ is an algebra isomorphism from $L^{1}\left(\omega_{1}\right)$ onto $L^{1}\left(\omega_{2}\right)$ then it is continuous [5; Remark 3(a)].

In this paper all of the algebras $L^{1}(\omega)$ are radical, or equivalently $\lim _{t \rightarrow \infty} \omega(t)^{1 / t}=0$.

In the following proposition, $M(\omega)$ is as defined in [4].

Proposition 1. Suppose $\theta$ is an isomorphism from $L^{1}\left(\omega_{1}\right)$ onto $L^{1}\left(\omega_{2}\right)$. Then the formula $\bar{\theta}(\mu)(f)=\theta\left(\mu * \theta^{-1}(f)\right) \quad\left(\mu \in M\left(\omega_{1}\right), \quad f \in L^{1}\left(\omega_{2}\right)\right)$ defines a continuous isomorphism $\bar{\theta}: M\left(\omega_{1}\right) \rightarrow M\left(\omega_{2}\right)$ which extends $\theta$.

Proof. For every $\mu \in M\left(\omega_{1}\right)$, let $T_{\mu}: L^{1}\left(\omega_{2}\right) \rightarrow L^{1}\left(\omega_{2}\right)$ be defined by $T_{\mu}(f)=\theta\left(\mu * \theta^{-1}(f)\right)$ $\left(f \in L^{1}\left(\omega_{2}\right)\right)$. Then $T_{\mu}$ is obviously linear and we have

$$
\begin{aligned}
T_{\mu}(f * g)=\theta\left(\mu * \theta^{-1}(f * g)\right) & =\theta\left(\mu * \theta^{-1}(f) * \theta^{-1}(g)\right) \\
& =\theta\left(\mu * \theta^{-1}(f)\right) * g=T_{\mu}(f) * g .
\end{aligned}
$$


Thus, $T_{\mu}$ is a multiplier on $L^{1}\left(\omega_{2}\right)$. By an identification of the multiplier algebra of $L^{1}\left(\omega_{2}\right)$ with $M\left(\omega_{2}\right)$ [4; Theorem 1.4], there exists a measure, say $\bar{\theta}(\mu)$, in $M\left(\omega_{2}\right)$ such that $T_{\mu}(f)$ $=\bar{\theta}(\mu) * f\left(f \in L^{1}\left(\omega_{2}\right)\right)$. We prove that the map $\mu \rightarrow \bar{\theta}(\mu)$ is an extension of $\theta$ to an isomorphism from $M\left(\omega_{1}\right)$ onto $M\left(\omega_{2}\right)$. This map is obviously linear. Let $\mu, v \in M\left(\omega_{1}\right)$ and $f \in\left(L^{1}\left(\omega_{2}\right) \backslash\{0\}\right)$. Then

$$
\begin{aligned}
\bar{\theta}(\mu * v) * f & =\theta\left(\mu * v * \theta^{-1}(f)\right)=\theta\left(\mu * \theta^{-1} \theta\left(v * \theta^{-1}(f)\right)\right) \\
& =\theta\left(\mu * \theta^{-1}(\bar{\theta}(v) * f)=\bar{\theta}(\mu) * \bar{\theta}(v) * f\right.
\end{aligned}
$$

which together with Titchmarsh's convolution theorem implies $\bar{\theta}(\mu * v)=\bar{\theta}(\mu) * \bar{\theta}(v)$.

Let $\bar{\theta}(\mu)=0$. Then for every $f \in\left(L^{1}\left(\omega_{2}\right) \backslash\{0\}\right)$

$$
\theta\left(\mu * \theta^{-1}(f)\right)=\bar{\theta}(\mu) * f=0,
$$

whence $\mu * \theta^{-1}(f)=0$, since $\theta$ is an isomorphism. Hence by Titchmarsh's convolution theorem $\mu=0$. Thus $\theta$ is injective.

To show that $\theta$ is onto, let $\mu \in M\left(\omega_{2}\right)$, then $f \rightarrow \theta^{-1}(\mu * \theta(f))$ is a multiplier on $L^{1}\left(\omega_{1}\right)$, whence there exists $v \in M\left(\omega_{1}\right)$ such that $\theta^{-1}(\mu * \theta(f))=v * f\left(f \in L^{1}\left(\omega_{1}\right)\right)$. If we apply $\bar{\theta}$ to both sides of this equality we obtain $\mu * \theta(f)=\bar{\theta}(v) * \theta(f) \quad\left(f \in L^{1}\left(\omega_{1}\right)\right)$. Another application of the Titchmarsh's convolution theorem implies $\mu=\bar{\theta}(v)$. It is easily verified that $\bar{\theta}$ extends $\theta$.

We also note that $(\bar{\theta})^{-1}=\overline{\left(\theta^{-1}\right)}$.

Lemma 1. Suppose $\theta$ is an isomorphism from $L^{1}\left(\omega_{1}\right)$ onto $L^{1}\left(\omega_{2}\right)$ and $\bar{\theta}$ is its extension as described in Proposition 1. Then there exists a constant $A_{\theta}>0$, such that

$$
\alpha\left(\bar{\theta}\left(\delta_{x}\right)\right)=A_{\theta} x \quad\left(x \in R^{+}\right)
$$

where $\delta_{x}$ is the unit mass concentrated at $x$.

Proof. We consider the function $\beta: R^{+} \rightarrow R^{+}$defined by $\beta(x)=\alpha\left[\bar{\theta}\left(\delta_{x}\right)\right]$. For every $x, y \in R^{+}$, by Titchmarsh's convolution theorem we have,

$$
\begin{aligned}
\beta(x+y) & =\alpha\left[\bar{\theta}\left(\delta_{x+y}\right)\right]=\alpha\left[\bar{\theta}\left(\delta_{x}\right) * \bar{\theta}\left(\delta_{y}\right)\right] \\
& =\alpha\left[\bar{\theta}\left(\delta_{x}\right)\right]+\alpha\left[\bar{\theta}\left(\delta_{y}\right)\right]=\beta(x)+\beta(y)
\end{aligned}
$$

Next we prove that $\beta$ is continuous from the right at every $x \in R^{+}$. It suffices to do this for $x=0$. Let $x_{n}>0$ and $x_{n} \rightarrow 0$. Then $\delta_{x_{n}} \stackrel{b s o}{\longrightarrow} \delta_{0}$. (For the definition of the topology bso and the topology $\sigma$ which follows, see [4].) Since $\bar{\theta}$ is an isomorphism from $M\left(\omega_{1}\right)$ onto $M\left(\omega_{2}\right)$ we have

$$
\bar{\theta}\left(\delta_{x_{n}} \stackrel{\text { bso }}{\longrightarrow} \bar{\theta}\left(\delta_{0}\right)=\delta_{0}\right.
$$


whence

$$
\bar{\theta}\left(\delta_{x_{n}} \stackrel{\sigma}{\longrightarrow} \delta_{0}\right.
$$

[4; Lemma 1.2]. This implies $\beta\left(x_{n}\right)=\alpha \bar{\theta}\left(\delta_{x_{n}}\right) \rightarrow 0$, for otherwise there exists a positive number $b$ such that for infinitely many values of $n, \alpha \bar{\theta}\left(\beta_{x_{n}}\right)>b$. Then if $f$ is a continuous function with supp $f \subset[0, b]$ and $f(0)=1$ we get $\int_{0}^{\infty} f(t) d \bar{\theta}\left(\delta_{x_{n}}\right)(t)=0$ for infinitely many values of $n$, while $\int_{0}^{\infty} f(t) d \delta_{0}(t)=f(0)=1$, and this contradicts (3). Hence $\beta$ is continuous from the right, whence there exists $A_{\theta} \geqq 0$ such that $\alpha\left(\bar{\theta}\left(\delta_{x}\right)\right)=\beta(x)=A_{\theta} x$ for every $x \in R^{+}$.

Next we prove that $A_{\theta}>0$. If $A_{\theta}=0$, then $\alpha\left(\bar{\theta}\left(\delta_{x}\right)\right)=0$ for every $x \in R^{+}$. We prove that this implies $\alpha(\theta(f))=0$ for every $f \in L^{1}\left(\omega_{1}\right)$ having a compact support and with $\alpha(f)>0$. Suppose $f \in L^{\mathbf{1}}\left(\omega_{1}\right)$, with $\alpha(f)=a$, supp $f \subset[a, b]$ where $0<a<b<\infty$. Then if $g=f * \delta_{-a}$, we have $g \in L^{1}\left(\omega_{1}\right), \alpha(g)=0$ and supp $g \subset[0, b-a]$. Therefore, $L^{1}\left(\omega_{1}\right) * g$ is dense in $L^{1}\left(\omega_{1}\right)$ [1; Theorem 2]. Since $\theta$ is an isomorphism between $L^{1}\left(\omega_{1}\right)$ and $L^{1}\left(\omega_{2}\right), L^{1}\left(\omega_{2}\right) * \theta(g)$ is dense in $L^{1}\left(\omega_{2}\right)$, whence $\alpha(\theta(g))=0$. We have $f=g * \delta_{a}$. Hence $\theta(f)=\theta(g) * \bar{\theta}\left(\delta_{a}\right)$. Thus,

$$
\alpha(\theta(f))=\alpha(\theta(g))+\alpha\left(\bar{\theta}\left(\delta_{a}\right)\right)=0 .
$$

To obtain a contradiction we show that there exists $f \in L^{1}\left(\omega_{1}\right)$ having a compact support with $\alpha(f)>0$ and with $\alpha(\theta(f))>0$.

There exists $K \geqq 1$ and $M>0$ such that

$$
\frac{\omega_{1}(K n)}{\omega_{2}(n)} \leqq M \quad(n \in N)
$$

[2; Theorem 4.1]. Since for each $\delta>0,\left(1 / \omega_{1}(\delta n)\right)^{1 / n} \rightarrow \infty$ as $n \rightarrow \infty$, by [2; Theorem 3.2.II] there exists $f$ in $L^{1}\left(\omega_{1}\right)$ with $\alpha(f)=K$, with supp $f \subset[K, K+1]$, and such that

$$
\left\|f^{* n}\right\|<\omega_{1}(K n) \quad(n \in N) .
$$

For this $f$, by (4) and (5) we have

$$
\left\|(\theta f)^{*_{n}}\right\| / \omega_{2}(n) \leqq M\|\theta\| \quad(n \in N)
$$

and so by [2; Theorem 3.6], $\alpha \theta(f) \geqq 1$. From this contradiction we conclude that $A_{\theta}>0$ and the lemma is proved.

The following proposition strengthens the statement of our Lemma 1.

Proposition 2. Suppose $\theta$ is an isomorphism from $L^{1}\left(\omega_{1}\right)$ onto $L^{1}\left(\omega_{2}\right)$ and $\bar{\theta}$ is its extension as described in Proposition 1. Then there exists a constant $A_{\theta}>0$, such that $\alpha(\bar{\theta}(\mu))=A_{\theta} \alpha(\mu)$, for every $\mu \in M\left(\omega_{1}\right)$.

Proof. By Lemma 1 there exists $A_{\theta}>0$ such that

$$
\alpha\left(\bar{\theta}\left(\delta_{x}\right)\right)=A_{\theta} x \quad\left(x \in R^{+}\right)
$$


Suppose

$$
\mu=\sum_{i=1}^{N} a_{i} \delta_{x_{i}}
$$

where $x_{1}<\ldots<x_{N}$ and $a_{i} \neq 0, i=1, \ldots, N$. Then

$$
\bar{\theta}(\mu)=\sum_{i=1}^{N} a_{i} \bar{\theta}\left(\delta_{x_{i}}\right)
$$

and we have

$$
\alpha \bar{\theta}\left(\delta_{x_{1}}\right)=A_{\theta} x_{1}<\ldots<\alpha \bar{\theta}\left(\delta_{x_{N}}\right)=A_{\theta} x_{N}
$$

Hence,

$$
\alpha(\bar{\theta}(\mu))=A_{\theta} x_{1}=A_{\theta} \alpha(\mu)
$$

For a general $\mu \in M\left(\omega_{1}\right)$, we first prove that $\alpha(\bar{\theta}(\mu)) \geqq A_{\theta} \alpha(\mu)$. Let $\left(\mu_{i}\right) \subset M\left(\omega_{1}\right)$ be a net such that $\mu_{i} \stackrel{b s o}{\longrightarrow} \mu, \alpha\left(\mu_{i}\right) \geqq \alpha(\mu)$ and such that each $\mu_{i}$ is a finite linear combination of point masses [4; Lemma 1.3]. Since $\bar{\theta}$ is an isomorphism we have $\bar{\theta}\left(\mu_{i}\right) \stackrel{b s o}{\longrightarrow} \bar{\theta}(\mu)$, whence $\bar{\theta}\left(\mu_{i}\right) \stackrel{\sigma}{\rightarrow} \bar{\theta}(\mu), \quad\left[4 ; \quad\right.$ Lemma 1.2]. If $\alpha(\bar{\theta}(\mu))<A_{\theta} \alpha(\mu)$, then we choose $b$ such that $\alpha \bar{\theta}(\mu)<b<A_{\theta} \alpha(\mu)$ and we let $g$ be a continuous function with supp $g \subset[\alpha \bar{\theta}(\mu), b]$ and with $\int_{0}^{\infty} g(x) d \bar{\theta}(\mu)(x) \neq 0$. Since $A_{\theta} \alpha(\mu) \leqq \alpha \bar{\theta}\left(\mu_{i}\right)$, we have $\int_{0}^{\infty} g(x) d \bar{\theta}\left(\mu_{i}\right)(x)=0$. Then

$$
0 \neq \int_{0}^{\infty} g(x) d \bar{\theta}(\mu)(x)=\lim \int_{0}^{\infty} g(x) d \bar{\theta}\left(\mu_{i}\right)(x)=0 .
$$

From this contradiction we conclude

$$
\alpha \bar{\theta}(\mu) \geqq A_{\theta} \alpha(\mu)
$$

Now, let $f \in L^{1}\left(\omega_{1}\right)$ have compact support and let $\alpha(f)=a$. Then $h=f * \delta_{-a} \in L^{1}\left(\omega_{1}\right)$, and $\alpha(h)=0$. Thus, $L^{1}\left(\omega_{1}\right) * h$ is dense in $L^{1}\left(\omega_{1}\right)$ [1; Theorem 2]. Hence, $L^{1}\left(\omega_{2}\right) * \theta(h)$ is dense in $L^{1}\left(\omega_{2}\right)$. Therefore, $\alpha \theta(h)=0$. We have $\theta(f)=\theta\left(h * \delta_{a}\right)=\theta(h) * \bar{\theta}\left(\delta_{a}\right)$. Hence,

$$
\alpha(\theta(f))=\alpha(\theta(h))+\alpha\left(\bar{\theta}\left(\delta_{a}\right)\right)=0+A_{\theta} \alpha=A_{\theta} \alpha(h) .
$$

Now, suppose $f \in\left(L^{1}\left(\omega_{1}\right) \backslash\{0\}\right), \alpha(f)=c$. Let $f_{1}=f \chi_{[c, c+1]}, f_{2}=f \chi_{(c+1, \infty)}$. Then $f=$ $f_{1}+f_{2}$. Therefore, $\theta(f)=\theta\left(f_{1}\right)+\theta\left(f_{2}\right)$. By the conclusion of the previous paragraph we have $\alpha \theta\left(f_{1}\right)=A_{\theta} c$, and by (2) we have $\alpha \theta\left(f_{2}\right) \geqq A_{\theta}(c+1)>A_{\theta} c$. Therefore

$$
\alpha(\theta(f))=\min \left\{\alpha\left(\theta\left(f_{1}\right)\right), \alpha\left(\theta\left(f_{2}\right)\right)\right\}=A_{\theta} c=A_{\theta} \alpha(f) .
$$

Finally, if $\mu \in\left(M\left(\omega_{1}\right) \backslash\{0\}\right)$, then for $f \in\left(L^{1}\left(\omega_{1}\right) \backslash\{0\}\right)$ by (4) we have

$$
\alpha(\bar{\theta}(\mu))+\alpha(\theta(f))=\alpha(\theta(\mu * f))=A_{\theta} \alpha(\mu * f)=A_{\theta} \alpha(\mu)+A_{\theta} \alpha(f) .
$$


Cancelling $\alpha \theta(f)=A_{\theta} \alpha(f)$ from both sides of (5) we obtain $\alpha \theta(\mu)=A_{\theta} \alpha(\mu)$, and the proof is complete.

Corollary 1. If $\theta$ is an isomorphism from $L^{1}\left(\omega_{1}\right)$ onto $L^{1}\left(\omega_{2}\right)$ and $\theta^{-1}$ is its inverse, then $A_{\theta-1}=1 / A_{\theta}$.

Proof. We have $\alpha\left(\bar{\theta}(\bar{\theta})^{-1}\left(\delta_{x}\right)\right)=\alpha\left(\delta_{x}\right)=x$, and by Proposition 2 we have $\alpha\left(\bar{\theta}(\bar{\theta})^{-1}\left(\delta_{x}\right)\right)$ $=A_{\theta} \alpha\left((\bar{\theta})^{-1}\left(\delta_{x}\right)\right)=A_{\theta} A_{\theta-1} x$, and the result follows.

Since $A_{\theta}>0$, we also have:

Corollary 2. The function $\alpha \bar{\theta}$ is strictly increasing in the sense that if $\alpha(\mu)<\alpha(v)$, then $\alpha(\bar{\theta}(\mu))<\alpha(\bar{\theta}(v))$.

We need the following two lemmas for the proof of our main theorem.

Lemma 2. Suppose that $\mu$ and $v$ are any two locally finite measures on $R^{+}$. Then $\mu * v$ has a non-zero mass at $\alpha(\mu * v)$ if and only if $\mu$ has a non-zero mass at $\alpha(\mu)$ and $v$ has $a$ non-zero mass at $\alpha(v)$.

Proof. We have $(\mu * v)(\{\alpha(\mu * v)\})=\mu(\{\alpha(\mu)\}) v(\{\alpha(v)\})$, and the lemma is proved.

Lemma 3. Suppose $\theta$ is an isomorphism from $L^{1}\left(\omega_{1}\right)$ onto $L^{1}\left(\omega_{2}\right)$ and $\bar{\theta}$ is its extension as described in Propositon 1. Then for every $x \in R^{+}, \bar{\theta}\left(\delta_{x}\right)$ has a non-zero mass at $\alpha\left(\bar{\theta}\left(\delta_{x}\right)\right)$.

Proof. Let $x \in R^{+}$and suppose that $\bar{\theta}\left(\delta_{x}\right)$ has a zero mass at $\alpha\left(\bar{\theta}\left(\delta_{x}\right)\right)$. Then, we first prove that $\bar{\theta}\left(\delta_{y}\right)$ has a zero mass at $\alpha\left(\bar{\theta}\left(\delta_{y}\right)\right)$, for every $y>0$. If $y>x$, then $\bar{\theta}\left(\delta_{y}\right)$ $=\bar{\theta}\left(\delta_{x}\right) * \bar{\theta}\left(\delta_{y-x}\right)$, whence by Lemma $2, \bar{\theta}\left(\delta_{y}\right)$ has a zero mass at $\alpha\left(\bar{\theta}\left(\delta_{y}\right)\right)$. On the other hand, if $0<y<x$, let $n$ be a positive integer such that $x<n y$. Then $\bar{\theta}\left(\delta_{n y}\right)$ $=\bar{\theta}\left(\delta_{n y-x}\right) * \bar{\theta}\left(\delta_{x}\right)$, and again Lemma 2 implies that $\theta\left(\delta_{n y}\right)$ has a zero mass at $\alpha\left(\bar{\theta}\left(\delta_{n y}\right)\right)$. Since $\bar{\theta}\left(\delta_{n y}\right)=\left(\bar{\theta}\left(\delta_{y}\right)\right)^{*_{n}}$, another application of Lemma 2 implies that $\bar{\theta}\left(\delta_{y}\right)$ has a zero mass at $\alpha\left(\bar{\theta}\left(\delta_{y}\right)\right)$.

Next we prove that this implies $\bar{\theta}(\mu)$ has a zero mass at $\alpha(\bar{\theta}(\mu))$, for every $\mu \in M\left(\omega_{1}\right)$ having a compact support and with $\alpha(\mu)>0$. Let $\mu \in M\left(\omega_{1}\right)$ with $\operatorname{supp} \mu \subset[a, b]$, $0<a<b<\infty$. Then $v=\mu * \beta_{-a / 2} \in M\left(\omega_{1}\right)$. We have $\mu=v * \delta_{a / 2}$, whence $\bar{\theta}(\mu)=\bar{\theta}(v) * \bar{\theta}\left(\delta_{a / 2}\right)$, and the discussion in the above paragraph together with Lemma 2 implies that $\bar{\theta}(\mu)$ has a zero mass at $\alpha(\bar{\theta}(\mu))$.

If $\lambda \in M\left(\omega_{1}\right)$ does not have compact support and $\alpha(\lambda)=k>0$, then we decompose $\lambda$ into $\lambda=\lambda_{1}+\lambda_{2}$, with supp $\lambda_{1} \subset[k, k+1], \alpha\left(\lambda_{1}\right)=k$, and $\alpha\left(\lambda_{2}\right) \geqq k+1$, so that $\bar{\theta}\left(\lambda_{1}\right)$ has a zero mass at $\alpha\left(\bar{\theta}\left(\lambda_{1}\right)\right)$. Since $\alpha\left(\bar{\theta}\left(\lambda_{2}\right)\right) \geqq A_{\theta}(k+1)>A_{\theta} k=\alpha\left(\bar{\theta}\left(\lambda_{1}\right)\right)$, the measure $\bar{\theta}(\lambda)=\bar{\theta}\left(\lambda_{1}\right)$ $+\bar{\theta}\left(\lambda_{2}\right)$, has a zero mass at $\alpha(\bar{\theta}(\lambda))=\alpha\left(\bar{\theta}\left(\lambda_{1}\right)\right)$.

Now, $\alpha\left((\bar{\theta})^{-1}\left(\delta_{1}\right)\right)=A_{\theta-1}>0$. Hence $\delta_{1}=\bar{\theta}(\bar{\theta})^{-1}\left(\delta_{1}\right)$ has a zero mass at $\alpha\left(\left(\bar{\theta}(\bar{\theta})^{-1}\right)\left(\delta_{1}\right)\right)$ $=\alpha\left(\delta_{1}\right)=1$. From this contradiction we conclude that $\dot{\theta}\left(\delta_{x}\right)$ has a non-zero mass at $\alpha\left(\bar{\theta}\left(\delta_{x}\right)\right)$ for every $x \in R^{+}$. 
Theorem 1. A necessary and sufficient condition for $L^{1}\left(\omega_{1}\right)$ and $L^{1}\left(\omega_{2}\right)$ to be isomorphic is the existence of positive numbers $a, b, m$ and $M$ such that,

$$
m \leqq \frac{\omega_{2}(a x)}{\omega_{1}(x)} b^{x} \leqq M \quad\left(x \in R^{+}\right) .
$$

Proof. Suppose that there exist $a>0, b>0, m>0$ and $M>0$ for which (1) is fulfilled. Define $\theta: L^{1}\left(\omega_{1}\right) \rightarrow L^{1}\left(\omega_{2}\right)$ by

$$
(\theta f)(x)=1 / a b^{x / a} f(x / a) \quad\left(f \in L^{1}\left(\omega_{1}\right), \quad \text { a.e. } \quad x \in R^{+}\right) .
$$

Then $\theta$ is an isomorphism from $L^{1}\left(\omega_{1}\right)$ onto $L^{1}\left(\omega_{2}\right)$.

Conversely, let $\theta$ be an isomorphism from $L^{1}\left(\omega_{1}\right)$ onto $L^{1}\left(\omega_{2}\right)$ and let $\bar{\theta}$ be its extension. For simplicity we write $a$ for $\boldsymbol{A}_{\theta}$ which is in fact $\alpha\left(\bar{\theta}\left(\delta_{1}\right)\right)$. For every rational $x \in R^{+}$, by Proposition 2 and Lemma 3 we have

$$
\bar{\theta}\left(\delta_{x}\right)=k(x) \delta_{a x}+\mu_{x}
$$

where $\alpha\left(\mu_{x}\right) \geqq a x, \mu_{x}(\{a x\})=0$, and $k(x) \neq 0$. Now, suppose $x, y \in R^{+}$are any two rationals. Then

$$
\bar{\theta}\left(\delta_{x+y}\right)=k(x+y) \delta_{a(x+y)}+\mu_{x+y}
$$

Also,

$$
\begin{aligned}
\bar{\theta}\left(\delta_{x+y}\right) & =\bar{\theta}\left(\delta_{x}\right) * \bar{\theta}\left(\delta_{y}\right)=\left(k(x) \delta_{a x}+\mu_{x}\right) *\left(k(y) \delta_{a y}+\mu_{y}\right) \\
& =k(x) k(y) \delta_{a x+a y}+k(x) \delta_{a x} * \mu_{y}+k(y) \delta_{a y} * \mu_{x}+\mu_{x} * \mu_{y}
\end{aligned}
$$

where the measure $k(x) \delta_{a x} * \mu_{y}+k(y) \delta_{a y} * \mu_{x}+\mu_{x} * \mu_{y}$ has a zero mass at $a x+a y$, by Lemma 3. Comparing equations (4) and (5) we get

$$
k(x+y)=k(x) k(y)
$$

Therefore, there exists $b>0$, such that for every rational $x \in R^{+}$, we have $|k(x)|=b^{x}$.

From (3), for every rational $x \in R^{+}$, we get

$$
\begin{aligned}
\left\|\bar{\theta}\left(\delta_{x}\right)\right\|_{M\left(\omega_{2}\right)} & =\left\|k(x) \delta_{a x}\right\|_{M\left(\omega_{2}\right)}+\left\|\mu_{x}\right\|_{M\left(\omega_{2}\right)} \geqq\left\|k(x) \delta_{a x}\right\|_{M\left(\omega_{2}\right)} \\
& =|k(x)|\left\|\delta_{a x}\right\|_{M\left(\omega_{2}\right)}=b^{x} \omega_{2}(a x)
\end{aligned}
$$

Now,

$$
\|\bar{\theta}\| \geqq \frac{\left\|\bar{\theta}\left(\delta_{x}\right)\right\|_{M\left(\omega_{2}\right)}}{\left\|\delta_{x}\right\|_{M\left(\omega_{1}\right)}}=\frac{b^{x} \omega_{2}(a x)}{\omega_{1}(x)}
$$


On the other hand, by Corollary 1 and Lemma 3 we have,

$$
(\bar{\theta})^{-1}\left(\delta_{a x}\right)=l(x) \delta_{x}+v_{x},
$$

where $l(x) \neq 0, \alpha\left(v_{x}\right) \geqq x$ and $v_{x}$ has a zero mass at $x$. Arguing as we did in the previous paragraph, we find $c>0$ such that $|l(x)|=c^{x}$, for every rational $x \in R^{+}$. We prove $c=1 / b$, or equivalently $|k(1)||l(1)|=1$. We have

$$
\bar{\theta}\left(\delta_{1}\right)=k(1) \delta_{a}+\mu_{1}
$$

and,

$$
(\bar{\theta})^{-1}\left(\delta_{a}\right)=l(1) \delta_{1}+v_{1}
$$

where $\alpha\left(\mu_{1}\right) \geqq a, \mu_{1}(\{a\})=0$ and $\alpha\left(v_{1}\right) \geqq 1, v_{1}(\{1\})=0$. Let $\varepsilon>0$. Since the measure $\mu_{1}$ introduced in (10) has a zero mass at $a$ and is regular, there exists $\eta>0$, and a decomposition $\quad \mu_{1}=\mu_{1}^{\prime}+\mu_{1}^{\prime \prime}$, with $\operatorname{supp} \mu_{1}^{\prime} \subset[a, a+\eta], \quad \operatorname{supp} \mu_{1}^{\prime \prime} \subset[a+\eta, \infty)$ and $\left\|\mu_{1}^{\prime}\right\|_{M\left(\omega_{2}\right)}<\varepsilon$. Then from (10) and (11) we get,

$$
\begin{aligned}
\delta_{1} & =k(1)(\bar{\theta})^{-1}\left(\delta_{a}\right)+(\bar{\theta})^{-1}\left(\mu_{1}^{\prime}\right)+(\bar{\theta})^{-1}\left(\mu_{1}^{\prime \prime}\right) \\
& =k(1)\left(l(1) \delta_{1}+v_{1}\right)+(\bar{\theta})^{-1}\left(\mu_{1}^{\prime}\right)+(\bar{\theta})^{-1}\left(\mu_{1}^{\prime \prime}\right) \\
& =k(1) l(1) \delta_{1}+(\bar{\theta})^{-1}\left(\mu_{1}^{\prime}\right)+k(1) v_{1}+(\bar{\theta})^{-1}\left(\mu_{1}^{\prime \prime}\right) .
\end{aligned}
$$

The measure $(\bar{\theta})^{-1}\left(\mu_{1}^{\prime \prime}\right)$ has a zero mass at 1 , since

$$
\alpha\left((\bar{\theta})^{-1}\left(\mu_{1}^{\prime \prime}\right)\right)=A_{\theta-1} \alpha\left(\mu_{1}^{\prime \prime}\right) \geqq \frac{1}{a}(a+\eta)>1
$$

Also the measure $k(1) v_{1}$ has a zero mass at 1 , since $v_{1}$ already had this property. The measure $(\bar{\theta})^{-1}\left(\mu_{1}^{\prime}\right)$ might have a non-zero mass at 1 . Suppose

$$
(\bar{\theta})^{-1}\left(\mu_{1}^{\prime}\right)=p \delta_{1}+\lambda
$$

where $\alpha(\lambda) \geqq 1, \lambda(\{1\})=0$, From (12) and (13) we obtain

$$
\delta_{1}=k(1) l(1) \delta_{1}+\left(p \delta_{1}+\lambda\right)+k(1) v_{1}+(\bar{\theta})^{-1}\left(\mu_{1}^{\prime \prime}\right)
$$

On equating the coefficients of $\delta_{1}$ in both sides of (14) we obtain

$$
1=k(1) l(1)+p
$$

On the other hand, by (13) we have,

$$
\left\|(\bar{\theta})^{-1}\left(\mu_{1}^{\prime}\right)\right\|_{M\left(\omega_{1}\right)} \geqq\left\|p \delta_{1}\right\|_{M\left(\omega_{1}\right)}=|p| \omega_{1}(1),
$$


and,

$$
\left\|(\bar{\theta})^{-1}\left(\mu_{1}^{\prime}\right)\right\|_{M\left(\omega_{1}\right)} \leqq\left\|(\bar{\theta})^{-1}\right\|\left\|\mu_{1}^{\prime}\right\|_{M\left(\omega_{2}\right)} \leqq \varepsilon\left\|(\bar{\theta})^{-1}\right\| .
$$

From (16) and (17) we obtain

$$
|p| \leqq \frac{\left\|(\bar{\theta})^{-1}\right\|}{\omega_{1}(1)} \varepsilon
$$

Since $\varepsilon$ was arbitrary (15) and (18) imply

$$
1=k(1) l(1)
$$

Thus, $c=1 / b$, whence $l(x)=1 / b^{x}$, for every rational $x \in R^{+}$and by (9) for every rational $x \in R^{+}$, we have

$$
\frac{\omega_{1}(x)}{\omega_{2}(a x) b^{x}} \leqq\left\|(\bar{\theta})^{-1}\right\|
$$

Combining (8) and (20) we obtain

$$
\left\|(\bar{\theta})^{-1}\right\|^{-1} \leqq \frac{\omega_{2}(a x) b^{x}}{\omega_{1}(x)} \leqq\|\bar{\theta}\|
$$

for every rational $x \in R^{+}$. Now, continuity of $\omega_{1}$ and $\omega_{2}$ implies that (21) holds for every $x \in R^{+}$, and the proof is complete.

Corollary 3. Suppose $\theta$ is an automorphism of $L^{1}(\omega)$, then $\alpha(\theta(f))=\alpha(f)\left(f \in L^{1}(\omega)\right)$.

Proof. By Theorem 1 there exist $a>0, b>0, m>0$, and $M>0$ such that $m \leqq[\omega(a x) / \omega(x)] b^{x} \leqq M\left(x \in R^{+}\right)$. The proof of Theorem 1 shows that $a$ can be chosen to be equal to $A_{\theta}$. If $a>1$, then

$$
m \leqq \frac{\omega(a x)}{\omega(x)} b^{x} \leqq \frac{\omega((a-1) x) \omega(x)}{\omega(x)} b^{x}=\omega((a-1) x) b^{x},
$$

whence $m^{1 / x} \leqq(\omega((a-1) x))^{1 / x} b$. Now we let $x \rightarrow \infty$ to obtain $1 \leqq 0$, a contradiction. Similarly, the inequality $[\omega(a x) / \omega(x)] b^{x} \leqq M$, rules out the possibility $a<1$. Hence $A_{\theta}=a=1$.

\section{REFERENCES}

1. G. R. Allan, Ideals of rapidly growing functions, Proc. of Int. Symp. on Functional Analysis and Applications, Ibadan, Nigeria, 1977.

2. W. G. BADE and H. G. Dales, Norms and ideals in radical convolution algebras, $J$. Functional Analysis 41 (1981), 77-109, 
3. I. M. Gelfand, D. A. Raikov and G. E. Shilov, Commutative Normed Rings (Chelsea, New York, 1964).

4. F. Ghahramani, Homomorphisms and derivations on weighted convolution algebras, $J$. London Math. Soc. (2) 21 (1980), 149-161.

5. N. P. Jewell and A. M. Sinclair, Epimorphisms and derivations on $L^{1}(0,1)$ are continuous, Bull. London Math. Soc. 8 (1976), 135-139.

Department of Mathematics

University for Teacher Education

49 Mofateh Ave.

Tehran

IrAN 